\title{
Risk factors for fistula formation after interstitial brachytherapy for locally advanced gynecological cancers involving vagina
}

\author{
Allen Yen, BS, Zhen Tian, PhD, Brian Hrycushko, PhD, Kevin Albuquerque, MD, MS \\ Department of Radiation Oncology, University of Texas Southwestern Medical Center, Dallas, TX, USA
}

\begin{abstract}
Purpose: To determine risk factors for fistula formation after interstitial brachytherapy (ISBT) in patients with advanced gynecologic cancers.

Material and methods: We performed an Institutional Review Board (IRB) approved retrospective review of 44 patients treated with transperineal template-based ISBT from 2011 to 2017 at a major metropolitan county and university health system. All patients were treated with image-guided high-dose-rate ISBT. Statistical analysis was performed using the $\chi^{2}$ test to identify factors correlated with fistula formation. Survival and tumor control outcomes were calculated using Kaplan Meier analyses.

Results: Patients had a mean age of 53 years (range, 28-81 years), a mean external beam dose of 43.1 Gy (range, 42.5$51.3 \mathrm{~Gy}$ ), and a mean brachytherapy dose of 22.8 Gy (range, 21.3-30 Gy). Two of 44 patients had fistulas that could be definitively attributed to therapy for a fistula rate of $4.5 \%$. Six additional patients $(13.6 \%)$ developed fistula after treatment with associated recurrent disease but were included in the causality analysis. We analyzed patient tumor and treatment factors, and on univariate analyses we found that age $\geq 60$ years, Hispanic ethnicity, bladder involvement, rectal D2 cc $\geq 70 \mathrm{~Gy}$, and whether patients had post-radiation biopsies were predictors for fistula formation. The 1-year overall survival (OS), progression-free survival (PFS), and local control (LC) were $85 \%, 58.5 \%$, and 76.9\%, respectively, with a mean follow-up time 23 months (range, 4.0-68.8 months).

Conclusions: We identified factors that predict fistula formation in patients with advanced gynecologic tumors treated with ISBT. These factors can be used to stratify patients into a high-risk group, with potential for modification of brachytherapy planning to reduce their risk of fistula formation.
\end{abstract}

J Contemp Brachytherapy 2018; 10, 6: 510-515 DOI: https://doi.org/10.5114/jcb.2018.80171

Key words: brachytherapy, fistula, vagina cancer.

\section{Purpose}

Radiation is an essential modality of therapy that is used to treat patients with locally advanced gynecologic (GYN) cancers. Patients are typically treated with pelvic chemoradiation therapy followed by highdose-rate (HDR) brachytherapy [1,2,3,4]. For these patients, brachytherapy is an important part of their treatment and can improve patient outcomes [5,6]. Normally, these patients are treated with intracavitary brachytherapy, where the applicator is placed directly into the cervix. However, for patients with larger tumors with distal vaginal involvement, bulky tumors with poor response, vaginal stenosis, which does not permit intracavitary treatment, or recurrent disease, interstitial brachytherapy (ISBT) is necessary for satisfactory coverage $[7,8,9]$. In ISBT, a transperineal template is used through which several hollow needles are directly inserted into the tissue to deliver dose more selectively to the tumor [10].

Studies have shown that interstitial brachytherapy is highly effective for locally advanced gynecologic cancers, with a 2-year locoregional control ranging from 51.3\% to $93 \%[11,12]$. Another study found that ISBT had similar overall survival and local control when compared to intracavitary brachytherapy despite the ISBT group including patients with larger tumors [13]. Unfortunately, patients who are treated with interstitial brachytherapy are typically treated with a high-dose to a large volume of tissue, which increases the risk of delayed toxicity [14,15]. These toxicities can prolong recovery and could potentially be fatal. In particular, vesicovaginal or rectovaginal fistulas present a significant risk because they are extremely difficult to surgically repair and can significantly affect a patient's quality of life. Previous studies have shown
Address for correspondence: Kevin Albuquerque, MD, MS, Department of Radiation Oncology, University of Texas Southwestern Medical Center, 2280 Inwood Road, Dallas, TX 75390, USA, phone: +1 214645 8309, fax: +1 214645 8527, $\bowtie$ e-mail: Kevin.Albuquerque@UTSouthwestern.edu
Received: 02.09 .2018

Accepted: 10.11 .2018

Published: 28.12.2018 
that several factors including dose to $2 \mathrm{~cm}^{3}$ (D2 cc) of the rectum, smoking, comorbidities, and prior abdominal surgeries are predictive for fistula formation in patients treated with traditional intracavitary brachytherapy and interstitial brachytherapy $[16,17,18,19,20,21,22,23]$. However, risk factors specifically for fistula formation have not been studied in patients treated with interstitial brachytherapy due to small number of patients treated by this modality. The purpose of this study was to determine risk factors for fistula formation with ISBT. These risk factors could be used to identify patients at a high-risk for fistula formation and subsequently change clinical decisions and treatment planning to prevent toxicities.

\section{Material and methods}

\section{Patients}

After Institutional Review Board (IRB) approval, we analyzed patients treated with ISBT for GYN cancers from years 2011 to 2017 . We found 44 patients who had been treated with interstitial brachytherapy. Demographic information, treatment regiments, toxicities, health information, survival, and recurrence data were collected through a review of the electronic medical records. Patients had a variety of gynecologic cancers including vaginal, endometrial, and cervical cancer. Three patients included in the study had recurrent cancer and 3 were treated with radiation previously.

\section{Treatment}

All patients were treated with ISBT following ABS guidelines, with 3-dimensional CT-volumetric planning as previously described $[8,24]$. Patients were treated with one insertion and five BID fractions of 4.5 to 5.5 Gy each to the clinical target volume. CTV contours were determined based on fiducial marker, clinical extent at the time of implant, cross sectional CT imaging at the time of implant, and cognitive fusion with preimplant MRI if available.

\section{Statistical analysis}

Statistical analyses were performed using IBM SPSS Statistics, version 24 (IBM Corps). Descriptive statistics reported mean, median, range, and standard deviation. Local control (LC), regional control (RC), overall survival (OS), and progression-free survival (PFS) were estimated using the Kaplan-Meier method. LC and RC were calculated in months from the start of radiation therapy to the date at which tumor progression occurred. OS was calculated in months from the start of radiation therapy to the date of death from any cause or censorship, which was defined as the last date of contact. PFS was calculated from the start of therapy to any recurrence or death. Univariate analysis was analyzed with the $\chi^{2}$ test. Univariate analysis looked at numerous prognostic factors including bladder and rectum invasion, and whether patients had biopsies or not. Bladder and rectum invasion were confirmed through either imaging or biopsy. Biopsies were defined as a biopsy of the vagina or treatment site post-treatment. Lastly, fistula formation was defined as an abnormal connection between the vagina and the bladder, ureters, rectum, or sigmoid colon and confirmed through either radiologic imaging or clinical evidence. Univariate logistic regression and area under the curve analysis were also performed to verify our results. Multivariate analysis was performed through MANOVA. Statistical significance was determined as $p<0.05$.

\section{Toxicity}

Toxicities were graded based on the Common Terminology Criteria for Adverse Events version 5.0 developed by the National Cancer Institute.

\section{Results}

Forty-four patients were included in the study. Tables 1-3 describe patient characteristics, radiation treatments, and dosimetric data.

Based on univariate analyses, age $\geq 60$ years, Hispanic ethnicity, bladder involvement, rectal D2 cc $\geq 70$ Gy, and whether patients had post-radiation biopsies or not were predictors for fistula formation. These factors were

Table 1. Patient demographics and cancer characteristics

\begin{tabular}{|c|c|}
\hline \multicolumn{2}{|l|}{ Variables } \\
\hline Patients & 44 \\
\hline Mean age, years (range) & $53(28-81)$ \\
\hline \multicolumn{2}{|l|}{ Race, number of people (\%) } \\
\hline African American & $9(20.5)$ \\
\hline Hispanic & $13(29.5)$ \\
\hline Caucasian & $21(47.7)$ \\
\hline Asian & $1(2.3)$ \\
\hline Mean Charlson comorbidity index (range) & $7.4(6-12)$ \\
\hline \multicolumn{2}{|l|}{ FIGO clinical stage, number of patients (\%) } \\
\hline \multicolumn{2}{|l|}{ Vaginal } \\
\hline$\|$ & $4(9.1)$ \\
\hline III & $7(15.9)$ \\
\hline \multicolumn{2}{|l|}{ Endometrial } \\
\hline ॥ & $1(2.3)$ \\
\hline Recurrent & $1(2.3)$ \\
\hline \multicolumn{2}{|l|}{ Cervical } \\
\hline IIB & $11(25)$ \\
\hline IIIA & $5(11.4)$ \\
\hline $\mathrm{IIIB}$ & $9(20.5)$ \\
\hline IVA & $4(9.1)$ \\
\hline Recurrent & $2(4.6)$ \\
\hline
\end{tabular}

FIGO - International Federation of Gynecology and Obstetrics 
Table 2. External radiation treatment modality

\begin{tabular}{lc} 
Number treated with IMRT (\%) & $32(72.7)$ \\
\hline Number treated with 3D conformal (\%) & $9(20.5)$ \\
\hline Number treated with EBRT at OSH (\%) & $4(9.0)$ \\
\hline Number treated with only ISBT (\%) & $1(2.3)$ \\
\hline EBRT fractionation, Gy/fx & $1.8-2.0$
\end{tabular}

IMRT - intensity-modulated radiation therapy; EBRT - external beam radiation therapy; OSH - outside hospital; ISBT - interstitial brachytherapy; fx - fraction

Table 3. Radiation therapy treatment and dosimetric data

\begin{tabular}{lc} 
Mean treatment days (SD) & $52(13.3)$ \\
\hline Mean WPRT dose, Gy (SD) & $43.1(7.8)$ \\
\hline Mean brachytherapy dose, Gy (SD) & $22.8(4.3)$ \\
\hline Mean bladder D2 cc, Gy (SD) & $77.1(12.9)$ \\
\hline Mean rectum D2 cc, Gy (SD) & $66.9(6.0)$ \\
\hline Mean $\mathrm{D}_{90}$, Gy (SD) & $81.8(6.8)$
\end{tabular}

SD - standard deviation; WPRT - whole-pelvis radiotherapy; D2 CC - dose to $2 \mathrm{~cm}^{3} ; D_{90}$-dose to $90 \%$ of the CTV

confirmed through univariate logistic regression. Table 4 summarizes the $p$-values from the $\chi^{2}$ univariate analysis for fistula formation and the number of patients within each sub-group with fistula formation. For this analysis, we included patients who had fistula associated with recurrence and it is possible that recurrence may have been partially responsible in the etiology of these events. However, we analyzed many possible factors associated with fistula formation because we believe that some factors are independent of recurrence and can be used to help predict patients at a high-risk for fistula formation.

Median and mean follow-up time were 16.9 months and 23 months, respectively (range, 4.0-68.8 months). Table 5 and Figure 1 summarize the survival data including LC, RC, PFS, and OS.

Observed toxicities are summarized in Table 6. Of the 44 patients, $8(20.5 \%)$ had grade $\geq 3$ GI or GU toxicities. Nine $(20.5 \%)$ of these patients had fistula formation with 1 vesicovaginal fistulas, 6 rectovaginal fistulas, and 2 with both. One patient had a fistula as a complication due to a hematoma. Of the $8(18.1 \%)$ remaining patients, 6 had fistula formation with concurrent recurrence, thus only $2(4.5 \%)$ patients had fistula formation definitively due to radiation treatment; however, all 8 patients were used in the causality analysis. These patients were managed either surgically through options like loop colostomies or symptomatically with the hope of the fistulas resolving without surgery. Of the 9 patients with fistulas, 3 were managed medically and 6 surgically. Three of these patients had grade 2 fistulas and 6 patients had grade 3 fistulas.

\section{Discussion}

Patients with advanced GYN tumors may require template-based interstitial brachytherapy boost in addi-
Table 4. Statistical significance from univariate analysis of risk factors for fistula formation

Factor Number of patients with fistula formation

Age at irradiation
$<60$ years
$\geq 60$ years

Race 0.02

Hispanic 5

Non-Hispanic 3

External beam therapy dose

$<50$ Gy 4

$\geq 50$ Gy 3

Brachytherapy dose $\quad 0.20$

$<25$ Gy 0

$\geq 25 \mathrm{~Gy} \quad 7$

\begin{tabular}{ll}
\hline D2 cc to bladder \\
\hline 60 Gy & 0 \\
$\geq 60$ Gy & 6
\end{tabular}

D2 cc to rectum

0.02

$\begin{array}{ll}<70 \text { Gy } & 0 \\ \geq 70 \text { Gy } & 7\end{array}$

\begin{tabular}{ll}
\hline $\mathrm{BMI}$ & 0.35 \\
\hline$<30$
\end{tabular}

\begin{tabular}{lll}
\hline$<30$ & 7 & \\
\hline$\geq 30$ & 1 & \\
\hline Smoking & & 0.54 \\
\hline
\end{tabular}

\begin{tabular}{ll}
\hline No smoking & 4 \\
\hline Current smoking & 1 \\
\hline Past smoking & 3 \\
\hline Vaginal invasion
\end{tabular}

Vaginal invasion

\begin{tabular}{lll}
\hline No & 4 & \\
\hline Bladder invasion & & 0.01 \\
\hline Yes & 7 & \\
\hline No & 1 & \\
\hline
\end{tabular}

\begin{tabular}{ll}
\hline Rectum invasion & 0.13 \\
\hline
\end{tabular}

\begin{tabular}{lll}
\hline Yes & 8 & \\
\hline No & 0 & \\
\hline Tumor size & & 0.43 \\
\hline Biopsy & & 0.04 \\
\hline Yes & 8 & \\
\hline No & 0 & \\
\hline
\end{tabular}

Previous pelvic radiation $\quad 0.73$

\begin{tabular}{lll}
\hline Yes & 1 & \\
\hline No & 7 & \\
\hline Previous abdominal surgery & & 0.77 \\
\hline Yes & 6 & \\
\hline No & 2 & \\
\hline Charlson score & & 0.31
\end{tabular}

Charlson score 0.31 
tion to standard external beam radiation therapy, though in some cases, interstitial brachytherapy can be monotherapeutic. Although ISBT can improve outcomes, the increased dose can lead to a greater risk of complications. Few studies $[16,25]$ have examined predictors for severe toxicity following template-based ISBT for advanced GYN tumors. From our literature search, no studies have specifically looked at fistula formation after ISBT. This study attempted to define factors that lead to fistula formation for 44 patients with locally advanced gynecologic cancers treated with template-based HDR interstitial brachytherapy.

Our univariate analysis identified age $\geq 60$ years, Hispanic ethnicity, D2 cc to rectum $\geq 70 \mathrm{~Gy}$, bladder involve-

\section{A}

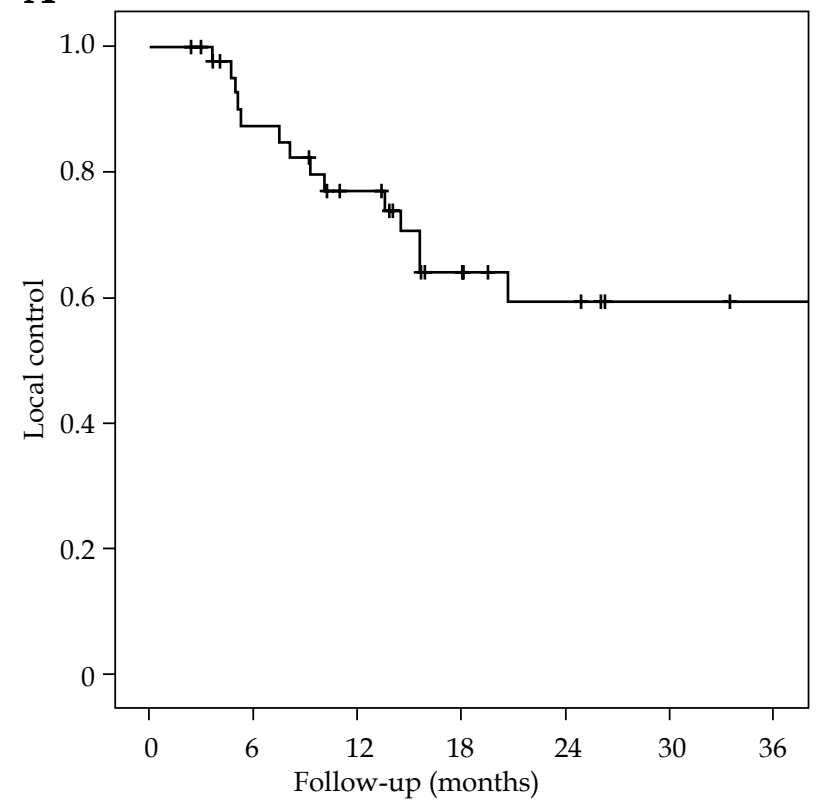

C

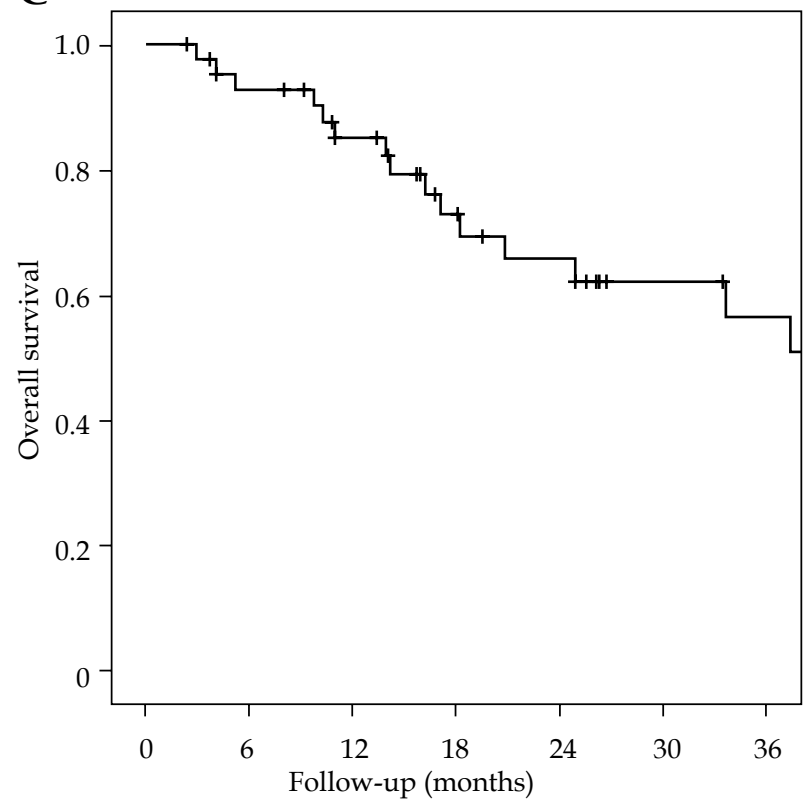

Table 5. Survival data

\begin{tabular}{lccc} 
Survival & Mean (months) & 1-year & 2-year \\
\hline LC & 43.5 & $76.90 \%$ & $59.30 \%$ \\
\hline RC & 55.8 & $81.40 \%$ & $78.20 \%$ \\
\hline OS & 43.1 & $85.00 \%$ & $62.00 \%$ \\
\hline PFS & 33.9 & $58.50 \%$ & $49.90 \%$
\end{tabular}

LC - local control; RC - regional control; OS - overall survival; PFS - progression-free survival

ment, and whether patients had post-radiation biopsies or not as predictors for fistula formation $(p=0.03,0.02$, $0.02,0.01,0.04$, respectively).

\section{B}

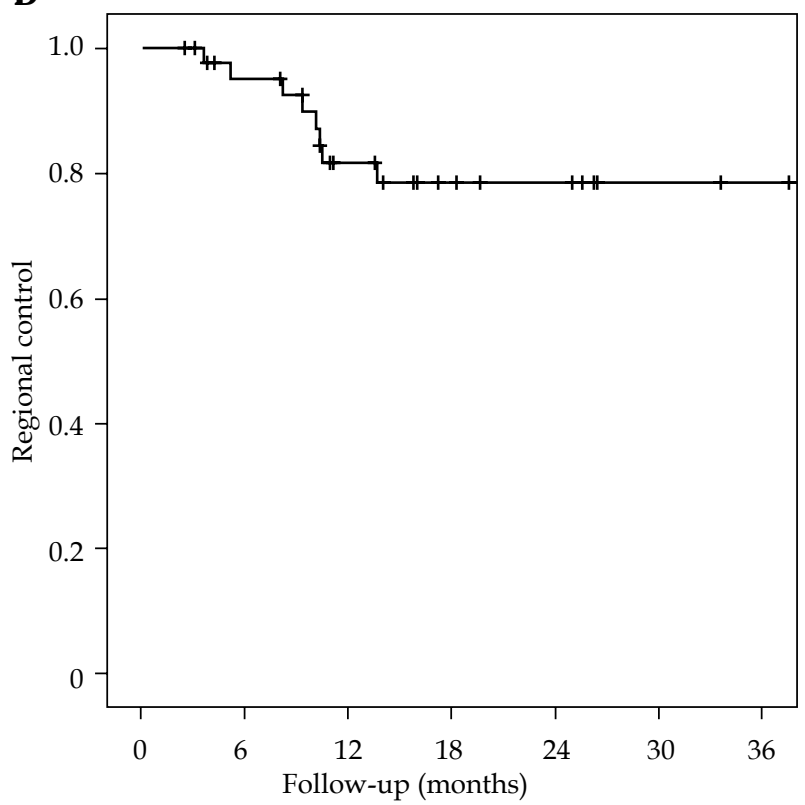

D

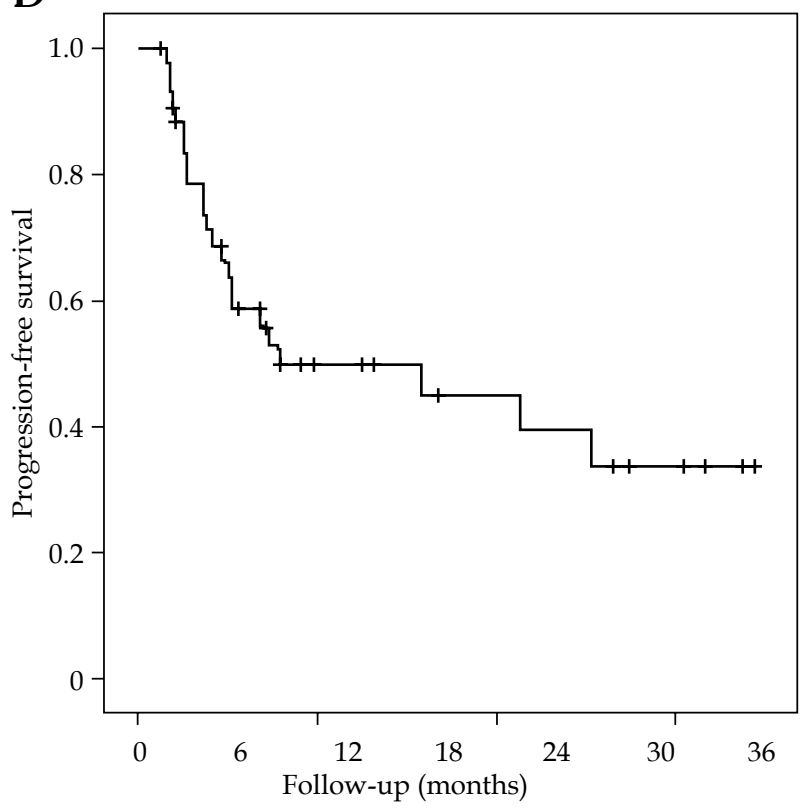

Fig. 1. A) Local control; B) Regional control; C) Overall survival; D) Progression-free survival 
Table 6. Patient toxicities

\begin{tabular}{lccc} 
Toxicity & Grade 0 & Grade $1-2$ & Grade 3-4 \\
\hline Gl & 26 & 10 & 8 \\
\hline GU & 36 & 5 & 3
\end{tabular}

GI - gastrointestinal; $G U$ - genitourinary

Although few studies have investigated predictors for fistula formation after ISBT, many studies have looked at predictors for fistula formation in traditional intracavitary brachytherapy and external beam radiotherapy. In contrast to our findings, Lebioda studied predictors of rectovaginal fistula formation in patients treated with LDR brachytherapy and found that age was not a predictor [19]. Similarly, Biewenga et al. found that age was not a predictor of vesicovaginal or rectovaginal fistula formation in patients with IVA cervical cancer [23]. Interestingly, another study that examined surgical treatment of rectovaginal fistulas postulated that age could affect tissue quality, which could lead to an increased risk of fistula formation [26].

Few studies have investigated race as a predictor for fistula formation. One study published in 1995 found that African American and Caucasian patients with stage IB cervical carcinomas treated with AP-PA radiation therapy were at higher risk for GI complications compared with Hispanic patients with the same stage of the disease and treatment [27]. There were no differences in weight or anterior-posterior separation between the groups. In contrary, our patient cohort found Hispanics more at risk for fistula formation in comparison to other races.

$\mathrm{D} 2 \mathrm{cc}$ to the rectum has been well established as a risk factor for GI toxicities and fistula formation. Lee and Viswanathan identified the D2 cc of the rectum as a predictor for rectal toxicities in patients treated with image-guided ISBT, similar to our study [16]. Georg et al. looked at many different parameters in dose-volume histograms and their abilities to predict late toxicities in patients treated with EBRT and brachytherapy. He identified D2 cc and D1 cc of the rectum as strong predictors for rectal toxicity [21]. Similarly, Kasibhatla et al. [16,25] studied predictors for GI toxicities in patients treated with EBRT and ISBT for advanced and recurrent GYN cancers. His study found that the 3-year risk of rectovaginal fistulas was significantly higher in patients who received a cumulative dose $>76$ Gy to the rectum. Similarly, the EMBRACE study found that patients with a D2 cc to the rectum $\geq 75$ Gy were at a $12.5 \%$ risk of fistula formation compared to $0-2.7 \%$ risk for lower doses [22]. We found the cut off rectal D2 cc dose that correlated with a significant incidence of fistula to be $70 \mathrm{~Gy}$, which is closer to what has been reported by Lee et al. at 72 Gy $[16,25]$. Like the previous authors, we also found D2 cc of the rectum to be a predictor for fistula formation, since the majority of patients with fistula had rectovaginal fistulas.

Several studies have shown that bladder involvement is a risk factor for fistula formation $[17,28,29,30]$. Sun et al. reported that for patients with locally advanced cervical cancer, anterior tumor necrosis from bladder invasion was associated with vesicovaginal fistula formation [28].
Additionally, Moore et al. studied patients with cervical cancer that had invaded the bladder and found that 11 out of 23 patients had vesicovaginal fistulas. They concluded that patients with stage IVA cervical cancer that had invaded the bladder were at an extremely high-risk for fistula formation [17]. Hata et al. hypothesized that this may be due to rapid reduction in tumor volume that leads to fistula formation [30]. From this perspective, vesicovaginal fistulas could be a result of tumor response to treatment. We also hypothesize that patients with bladder invasion have tumors more likely to be aggressive and consequently are treated with a higher dose of radiation, which in general could predispose to more toxicity.

Lastly, we identified one study that showed post-radiation biopsies to be associated with fistula formation. Feddock et al. performed a retrospective study on the impact of post-radiation biopsies on patients with cervical cancer. Of the 89 patients who underwent invasive biopsy, 9 subsequently developed fistula. It was concluded that post-radiation biopsy was a risk factor for fistula formation [31]. It is possible that post-radiation biopsies are indicators for recurrence. In this scenario, tumor recurrence, rather than the biopsy itself, could cause tissue damage, which could lead to fistula formation. On the other hand, the presence of radiation changes or necrosis, which appear similar to recurrence, may require biopsy causing further trauma precipitating a fistula.

Other studies have identified additional factors that increase the risk of fistula formation. Murakami et al. investigated gynecologic malignancies treated with ISBT and identified re-irradiation and vaginal D2 cc as predictors of vaginal ulcers, which could develop into fistulas [32]. Moore et al. found that smoking, bladder, and rectum invasion were associated with patients with fistula formation. $56.5 \%$ of the study population of Moore et al. was found to be smokers, compared to 7 out of 44 patients $(15.9 \%)$ in our study. This difference could explain why our study did not find smoking as a risk factor for fistula formation [17].

Limitations of our study include the retrospective nature of the study and the heterogeneity in the study population, since patients with primary and recurrent cervical, endometrial, and vaginal cancers were included and patients were not all treated with the same EBRT techniques. Additionally, the sample size was small, with 44 patients having had a limited follow-up period. Lastly, since only 8 of 44 patients had fistula included in analysis, the small sample size could impact the $\chi^{2}$ analysis. Regardless, we were able to identify some risk factors for fistula formation.

\section{Conclusions}

We identified prognostic factors that could predict fistula formation in gynecologic cancers after interstitial brachytherapy. In summary, the prognostic factors identified that predict fistula formation are age $\geq 60$ years, Hispanic ethnicity, D2 cc to rectum $\geq 70 \mathrm{~Gy}$, bladder involvement, and whether patients had post-radiation biopsies. Using these factors, we hope physicians will be able to identify patients at a higher risk for fistula formation af- 
ter ISBT and make potential planning changes to reduce toxicities. However, there continues to be a need to further study patients treated by this modality. Studying the same principles through a multi-institutional study with a larger patient population could help confirm the factors we have identified in this study and indicate other factors that could predict fistula formation. This study is one step towards an eventual goal of identifying risk factors for fistula formation in patients treated with interstitial brachytherapy.

\section{Disclosure}

Authors report no conflict of interest.

\section{References}

1. Marth C, Landoni F, Mahner S et al. Cervical cancer: ESMO Clinical Practice Guidelines for diagnosis, treatment and follow-up. Ann Oncol 2017; 28: 72-83.

2. Board, P.D.Q.A.T.E. Vaginal Cancer Treatment (PDQ(R)): Health Professional Version, in PDQ Cancer Information Summaries. National Cancer Institute (US): Bethesda (MD). 2002.

3. Board, P.D.Q.A.T.E. Endometrial Cancer Treatment (PD$\mathrm{Q}(\mathrm{R})$ ): Health Professional Version: in PDQ Cancer Information Summaries. National Cancer Institute (US): Bethesda (MD). 2002.

4. Glaser SM, Beriwal S. Brachytherapy for malignancies of the vagina in the 3D era. J Contemp Brachytherapy 2015; 7: 312-318.

5. Viswanathan AN, Cormack R, Rawal B, Lee H. Increasing brachytherapy dose predicts survival for interstitial and tandem-based radiation for stage IIIB cervical cancer. Int J Gynecol Cancer 2009; 19: 1402-1406.

6. Viswanathan AN, Moughan J, Small W Jr et al. The quality of cervical cancer brachytherapy implantation and the impact on local recurrence and disease-free survival in radiation therapy oncology group prospective trials 0116 and 0128 . Int I Gynecol Cancer 2012; 22: 123-131.

7. Monk BJ, Tewari K, Burger RA et al. A comparison of intracavitary versus interstitial irradiation in the treatment of cervical cancer. Gynecol Oncol 1997; 67: 241-247.

8. Beriwal S, Demanes DJ, Erickson B et al. American Brachytherapy Society consensus guidelines for interstitial brachytherapy for vaginal cancer. Brachytherapy 2012; 11: 68-75.

9. Kannan N, Beriwal S, Kim H et al. High-dose-rate interstitial computed tomography-based brachytherapy for the treatment of cervical cancer: Early results. Brachytherapy 2012; 11: 408-412.

10. Banerjee R, Kamrava M. Brachytherapy in the treatment of cervical cancer: a review. Int I Womens Health 2014; 6: 555-564.

11. Manuel M, Cho L, Catalano P et al. Outcomes with Image-based Interstitial Brachytherapy for Vaginal Cancer. $R a-$ diother Oncol 2016; 120: 486-492.

12. Umezawa R, Murakami N, Nakamura $S$ et al. Image-guided interstitial high-dose-rate brachytherapy for locally recurrent uterine cervical cancer: A single-institution study. Brachytherapy 2018; 17: 368-376.

13. Sekii S, Murakami N, Kato T et al. Outcomes of salvage highdose-rate brachytherapy with or without external beam radiotherapy for isolated vaginal recurrence of endometrial cancer. I Contemp Brachytherapy 2017; 9: 209-215.

14. Syed AM, Puthawala AA, Abdelaziz NN et al. Long-term results of low-dose-rate interstitial-intracavitary brachytherapy in the treatment of carcinoma of the cervix. Int J Radiat Oncol Biol Phys 2002; 54: 67-78.
15. Hughes-Davies L, Silver B, Kapp DS. Parametrial interstitial brachytherapy for advanced or recurrent pelvic malignancy: the Harvard/Stanford experience. Gynecol Oncol 1995; 58: 24-27.

16. Lee LJ, Viswanathan AN. Predictors of toxicity after imageguided high-dose-rate interstitial brachytherapy for gynecologic cancer. Int I Radiat Oncol Biol Phys 2012; 84: 1192-1197.

17. Moore KN, Gold MA, McMeekin DS et al. Vesicovaginal fistula formation in patients with Stage IVA cervical carcinoma. Gynecol Oncol 2007; 106: 498-501.

18. Denlinger N, Bixel NN, Salani K et al. Interstitial Brachytherapy for Locally Advanced Cervical Cancer. Int J Womens Health Wellness 2015; 1: 1.

19. Lebioda A. Rectovaginal fistula risk doses in patients with cervical cancer. Rep Pract Oncol Radiother 2004; 9: 37-43.

20. Coia LR, Myerson RJ, Tepper JE. Late effects of radiation therapy on the gastrointestinal tract. Int J Radiat Oncol Biol Phys 1995; 31: 1213-1236.

21. Georg P, Lang S, Dimopoulos JC et al. Dose-volume histogram parameters and late side effects in magnetic resonance image-guided adaptive cervical cancer brachytherapy. Int I Radiat Oncol Biol Phys 2011; 79: 356-362.

22. Mazeron R, Fokdal LU, Kirchheiner K et al. Dose-volume effect relationships for late rectal morbidity in patients treated with chemoradiation and MRI-guided adaptive brachytherapy for locally advanced cervical cancer: Results from the prospective multicenter EMBRACE study. Radiother Oncol 2016; 120: 412-419

23. Biewenga P, Mutsaerts MA, Stalpers LJ et al. Can we predict vesicovaginal or rectovaginal fistula formation in patients with stage IVA cervical cancer? Int J Gynecol Cancer 2010; 20: 471-475.

24. Viswanathan AN, Cormack R, Holloway CL et al. Magnetic resonance-guided interstitial therapy for vaginal recurrence of endometrial cancer. Int J Radiat Oncol Biol Phys 2006; 66: 91-99.

25. Kasibhatla M, Clough RW, Montana GS et al. Predictors of severe gastrointestinal toxicity after external beam radiotherapy and interstitial brachytherapy for advanced or recurrent gynecologic malignancies. Int J Radiat Oncol Biol Phys 2006; 65: 398-403.

26. Zelga P, Tchórzewski M, Zelga $\mathrm{M}$ et al. Radiation-induced rectovaginal fistulas in locally advanced gynaecological malignancies-new patients, old problem? Lagenbecks Arch Surg 2017; 402: 1079-1088.

27. Eifel PJ, Levenback C, Wharton JT, Oswald MJ. Time course and incidence of late complications in patients treated with radiation therapy for FIGO stage IB carcinoma of the uterine cervix. Int J Radiat Oncol Biol Phys 1995; 32: 1289-1300.

28. Sun R, Koubaa I, Limkin EJ et al. Locally advanced cervical cancer with bladder invasion: clinical outcomes and predictive factors for vesicovaginal fistulae. Oncotarget 2018; 9: 9299-9310.

29. Sun R, Mazeron R, Koubaa I et al. OC-0366: Cervical cancer with bladder invasion: outcomes and vesicovaginal fistula prognostic factors. Radiother Oncol 2017; 123: S196-S197.

30. Hata M, Koike I, Miyagi E et al. Radiation therapy for stage IVA uterine cervical cancer: treatment outcomes including prognostic factors and risk of vesicovaginal and rectovaginal fistulas. Oncotarget 2017; 8: 112855-112866.

31. Feddock J, Randall M, Kudrimoti M et al. Impact of post-radiation biopsies on development of fistulae in patients with cervical cancer. Gynecol Oncol 2014; 133: 263-267.

32. Murakami N, Kasamatsu T, Sumi M et al. Vaginal tolerance of CT based image-guided high-dose rate interstitial brachytherapy for gynecological malignancies. Radiat Oncol 2014; 9: 31 